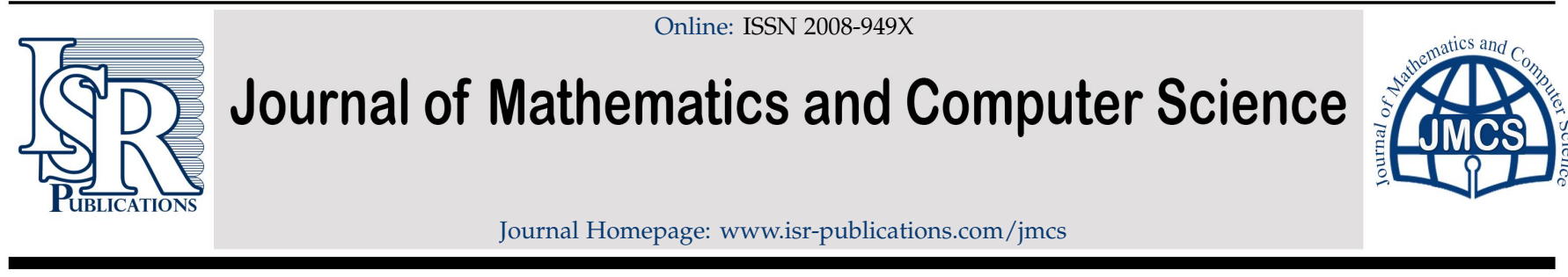

\title{
Sensitivity and optimal control analysis of HIV/AIDS model with two different stages of infection subpopulation
}

\author{
Ummu Habibah ${ }^{\mathrm{a}, *}$, Trisilowati ${ }^{\mathrm{a}}$, Yona Lotusia Pradana ${ }^{\mathrm{b}}$ \\ ${ }^{a}$ Department of Mathematics and member of Research Group of Biomathematics, Brawijaya University, Jl. Veteran Malang, Indonesia. \\ ${ }^{b}$ Undergraduate student of the Department of Mathematics, Brawijaya University, Jl. Veteran Malang, Indonesia.
}

\begin{abstract}
We study optimal control analysis of HIV/AIDS model with two different stages of infection subpopulation. There are two controls, antiretroviral (ARV) treatment is given to HIV-infected subpopulation, and HAART (highly active antiretroviral treatment) is given to full-blown AIDS subpopulation. Before that, we analyze the sensitivity of parameters which can be used to determine the most parameter take effect in the spread of HIV virus. We apply the sensitivity analysis to all the parameters appear in the reproduction number. Furthermore, we apply optimal control theory to minimize HIV-infected and full-blown AIDS subpopulation, and the cost related to the implementation of control strategies using the Pontryagin's Minimum Principle. We prove an existence of a optimal control pair. Numerical solution is conducted by solving the optimally system using the sweep backward and forward method. The results show that giving control pair in the model can decrease the infected and the full-blown AIDS subpopulations significantly.
\end{abstract}

Keywords: Optimal control, HIV / AIDS model, the Pontryagin's minimum principle.

2020 MSC: 46C05, 46C99, 25D15, 26D10.

(C)2022 All rights reserved.

\section{Introduction}

Acquired Immune Deficiency Syndrome (AIDS) is a disease caused by human immunodeficiency virus (HIV) that can suppress T-cells in the human body which has a function to against infections. The cells are important part of the body-immune system. AIDS becomes a major problem in the world because people infected with HIV are prone to various diseases which might lead to death. Some countries have attempted to reduce the growth rate of AIDS disease by implementing infection control programs including the use of condoms and sterile syringes [8].

Several mathematical model have been developed by researcher in order to understand the spread of HIV infection. Mathematical model with treatment where the population was divided into four compartments (susceptible (S), HIV infected (I), AIDS (A) and symptomatic (J) subpopulations) [3]. In 2014, the HIV / AIDS model was developed by adding the density of the dependency factor for infection [2]. Huo et al (2016) developed the HIV / AIDS epidemic model by adding the treatment stated in the SIATR model

\footnotetext{
*Corresponding author

Email address: ummu_habibah@ub.ac.id (Ummu Habibah)

doi: $10.22436 /$ jmcs.026.01.08
}

Received: 2020-08-12 Revised: 2021-07-30 Accepted: 2021-08-26 
and by analyzed dynamically [10]. Individuals in the T compartment received all types of treatment. In 2014, Huo and Chen conducted a dynamic analysis of the HIV/AIDS epidemic model with different stages of infection, namely positive individuals infected with HIV showing symptoms and without symptoms. After treatment, subpopulations with HIV symptoms will become HIV positive without symptoms. Ulfa et al. (2018) analyzed the dynamic model of HIV / AIDS with different stages of susceptible and infection subpopulations [20]. The susceptible subpopulation was divided into two, namely susceptible subpopulation that had knowledge about HIV / AIDS and susceptible individual that did not have knowledge about HIV / AIDS. The infected subpopulations were divided into two, the same as model in [9]. The results were local asymptotically stable with certain conditions for equilibrium points. [21] divided susceptible and infected populations into two, non-homosexual and homosexual susceptible subpopulations, and non-homosexual and homosexual HIV-infected subpopulations respectively. Recently, Habibah et al. developed the model with different stage of infection subpopulation motivated from above research. The dynamic of the models were analyzed dynamically. The result were endemic occurred when the basic reproduction number $R_{0}>1$ [8]. It is important to apply optimal control analysis in order to minimize HIV-infection subpopulation. Optimal control problems were studied in $[1,5,6,12,14,15,18]$. Subhas and Dibakar (2015) investigated a mathematical model depicting the non-linear dynamics of immunologic tumors and derive the existence and uniqueness of an optimal control by using the boundedness of solutions, and demonstrated the numerical simulations [11]. Habibah et al. (2018) developed the model of Huo et al. (2016) with two control strategies, an ARV and the highly active antiretroviral therapy (HAART) [7]. Unfortunately, they did not analyze the boundedness and positivity of solutions, and the importance is an existence of the optimal control in the system.

In this paper we study sensitivity and optimal control analysis of HIV/AIDS model. The sensitivity analysis of each parameter corresponds to the basic-reproduction number $R_{0}$ will be analyzed by calculating sensitivity index. The study of sensitivity analysis follows [4, 17]. We will find the most sensitive parameters to increase or decrease the basic reproduction number. Furthermore, we investigate HIV / AIDS epidemic model that was developed from the model of [8] by adding two control strategies, antiretroviral (ARV) treatment and HAART (highly active antiretroviral treatment). The optimal control theory is applied to handle the model. We prove existence of the optimal control pair. Optimally system is obtained by applying the Minimum-Pontryagin Principle in order to minimize HIV-infection and fullblown AIDS subpopulations, and the cost related to the treatments. Numerical solution is conducted by solving the optimally system using the sweep backward and forward method. The results showed that by giving ARV and HAART in the model can decrease the infected and the full-blown AIDS subpopulations significantly.

\section{The analysis of HIV/AIDS model}

Revisited [8], the mathematical model of HIV/AIDS with two different stages of infection subpopulation is

$$
\begin{aligned}
\dot{\mathrm{S}} & =\lambda-\beta_{1} \mathrm{SI}_{1}-\beta_{2} \mathrm{SI}_{2}-\mathrm{aS}, \\
\dot{\mathrm{I}_{1}} & =\beta_{1} \mathrm{SI}_{1}+\alpha_{1} \mathrm{~T}-\mathrm{bI}_{1}, \\
\dot{\mathrm{I}_{2}} & =\beta_{2} \mathrm{SI}_{2}-\mathrm{cI}_{2}, \\
\dot{\mathrm{A}} & =\mathrm{k}_{2} \mathrm{I}_{2}+\alpha_{2} \mathrm{~T}-\mathrm{eA}, \\
\dot{\mathrm{T}} & =\mathrm{k}_{1} \mathrm{I}_{1}+\mathrm{k}_{3} \mathrm{I}_{2}-\mathrm{fT}, \\
\dot{\mathrm{R}} & =\mu_{1} \mathrm{~S}-\mathrm{dR},
\end{aligned}
$$

with $a=\mu_{1}+d, b=k_{1}+d, c=k_{2}+k_{3}+d, e=\delta_{1}+d$, and $f=\alpha_{1}+\alpha_{2}+\delta_{2}+d$. The model has two equilibrium points, disease-free equilibrium point $X^{0}=\left(S^{0}, I_{1}^{0}, I_{2}^{0}, A^{0}, T^{0}, R^{0}\right)=\left(\frac{\lambda}{a}, 0,0,0,0, \frac{\mu_{1} \lambda}{d a}\right)$, and endemic equilibrium point

$$
X^{*}=\left(S^{*}, I_{1}^{*}, I_{2}^{*}, A^{*}, T^{*}, R^{*}\right),
$$


and

$$
\begin{aligned}
S^{*} & =\frac{c}{\beta_{2}}, \\
I_{1}^{*} & =\frac{\alpha_{1} k_{3}\left(\lambda \beta_{2}-a c\right)}{\alpha_{1} k_{3} \beta_{1} c+\beta_{2} b f c-\beta_{1} f c^{2}-c \alpha_{1} k_{1} \beta_{2}}=\frac{\left(\lambda \beta_{2}\right)}{a c}-1=R_{0}-1, \\
I_{2}^{*} & =\frac{\left(f \beta_{2} b-f \beta_{1} c-\alpha_{1} k_{1} \beta_{2}\right)}{\alpha_{1} k_{3} \beta_{2}} I_{1}^{*}, \\
A^{*} & =\frac{k_{2}\left(\beta_{2} b f-\beta_{1} c f-\alpha_{1} k_{1} \beta_{2}\right)+k_{3} \alpha_{2}\left(\beta_{2} b-\beta_{1} c\right)}{\alpha_{1} k_{3} \beta_{2} e} I_{1}^{*}, \\
T^{*} & =\frac{\beta_{2} b-\beta_{1} c}{\beta_{2} \alpha_{1}} I_{1}^{*}, \\
R^{*} & =\frac{\mu_{1} c}{\beta_{2} d}
\end{aligned}
$$

where the reproduction number is $R_{0}=\frac{\beta_{2} \lambda}{a c}$. Furthermore, the stability analysis result in the disease-free equilibrium $X^{0}$ of system (2.1) is said to be asymptotically stable if $R_{0}<1$ and vice versa is unstable, locally and globally.

\section{Sensitivity analysis of parameter}

Sensitivity analysis is used to identify the effect of each parameter on disease spread based on $R_{0}$, which can provide input in selecting appropriate control measures to prevent the spread of epidemic [17]. The sensitivity index can be calculated using following formula:

$$
C_{p}^{A}=\frac{\partial A}{\partial p} \frac{p}{A}
$$

where $A$ is differentiated function of a parameter $p$.

Table 1: Parameters and descriptions for simulations are adapted from [8].

\begin{tabular}{lll}
\hline Parameter & Description & Value \\
\hline$\lambda$ & Recruitment rate & 0.55 \\
$\beta_{1}$ & Transmision coefficient of $\mathrm{I}_{1}$ & 0.0023 \\
$\beta_{2}$ & Transmision coefficient of $\mathrm{I}_{2}$ & 0.0033 \\
$\alpha_{1}$ & The proportion of successful treatment & 0.02 \\
$\alpha_{2}$ & The proporsion of treatmen failure & 0.05 \\
$k_{1}$ & Progression rate from $\mathrm{I}_{1}$ to T & 0.0498 \\
$k_{2}$ & Progression rate from $\mathrm{I}_{2}$ to A & 0.008 \\
$k_{3}$ & Progression rate from $\mathrm{I}_{2}$ to T & 0.05 \\
$\mu_{1}$ & The rate of susceptible individuals who changed their habits & 0.03 \\
\hline
\end{tabular}

To reduce the number of deaths and illnesses caused by HIV infection, it is important to determine the cause of transmission of the virus. The importance of parameters for disease spread can be seen through this sensitivity index. Therefore, this method is used to determine the parameters that have a significant effect on $R_{0}$ and the parameters that need to be controlled. Parameters in the basic reproduction number $R_{0}$ are $\beta_{2}, \lambda, \mu_{1}, k_{2}, k_{3}, d$. Parameter sensitivity analysis is using the method in [5, 17]. Some of the 
parameters obtained are as follows:

$$
\begin{aligned}
& C_{\beta_{2}}^{R_{0}}=\frac{\partial R_{0}}{\partial \beta_{2}} \times \frac{\beta_{2}}{R_{0}}=1, \\
& C_{\lambda}^{R_{0}}=\frac{\partial R_{0}}{\partial \lambda} \times \frac{\lambda}{R_{0}}=1, \\
& C_{\mu_{1}}^{R_{0}}=\frac{\partial R_{0}}{\partial \mu_{1}} \times \frac{\mu_{1}}{R_{0}}=-\frac{\mu_{1}}{\mu_{1}+d}=-0.604839, \\
& C_{k_{2}}^{R_{0}}=\frac{\partial R_{0}}{\partial k_{2}} \times \frac{k_{2}}{R_{0}}=-\frac{k_{2}}{k_{2}+k_{3}+d}=-0.103093, \\
& C_{k_{3}}^{R_{0}}=\frac{\partial R_{0}}{\partial k_{3}} \times \frac{k_{3}}{R_{0}}=-\frac{k_{3}}{k_{2}+k_{3}+d}=-0.64433, \\
& C_{d}^{R_{0}}=\frac{\partial R_{0}}{\partial d} \times \frac{d}{R_{0}}=-\frac{d\left(k_{2}+k_{3}+2 d+\mu_{1}\right)}{\left(k_{2}+k_{3}+d\right)\left(\mu_{1}+d\right)}=-0.647739,
\end{aligned}
$$

based on the parameter values in Table 1 , it is obtained $R_{0}=0.471556$. Equations (3.1) show that the sensitivity index has positive and negative values. Positive value $\left(\lambda\right.$ and $\left.\beta_{2}\right)$ means if one of the parameter values is increased (decreased), then $R_{0}$ will increase (decrease). In this causes the spread of HIV/AIDS increases (decreases). Similar as positive value, sensitivity index has negative value $\left(\mu_{1}, d k_{2}\right.$, and $k_{3}$ ) means if one of these parameter values is increased (decreased), then $R_{0}$ will decrease (increase), therefore the spread of HIV/AIDS decrease (increase). When we take absolute values of the sensitivity index, the most sensitive parameters is recruitment rate $(\lambda)$ and the rate of transmission from uneducated subpopulations to infection not consuming $\operatorname{ARV}\left(\beta_{2}\right)$.

\section{Optimal control analysis}

We formulated optimal control problem applied to the HIV/AIDS model by Habibah et al. (2021) [8] since the endemic occurred when the basic reproduction number as a threshold of disease transmission was larger than one $R_{0}>1$. The formulated optimal control on the model can be written as follows

$$
\begin{aligned}
\dot{S} & =\lambda-\beta_{1} S_{1}-\beta_{2} S_{2}-a S, \\
I_{1} & =\beta_{1} S_{1} I_{1}+\alpha_{1} T-b I_{1}, \\
\dot{I_{2}} & =\beta_{2} S_{2}-c_{1} I_{2}-u_{1} I_{2}, \\
\dot{A} & =k_{2} I_{2}+\alpha_{2} T-e A-u_{2} A, \\
\dot{T} & =k_{1} I_{1}+u_{1} I_{2}-f T+u_{2} A, \\
\dot{R} & =\mu_{1} S-d R+u_{1} I_{2},
\end{aligned}
$$

with $a=\mu_{1}+d, b=k 1+d, c_{1}=k 2+d, e=d+\delta_{1}, f=\alpha_{1}+\alpha_{2}+d+\delta_{2}$.

We added two control strategies on the model. $k_{3}$ as a constant variable in [8] now becomes a function of time, we call it $u_{1}(t)$. Control $u_{1}(t)$ in this problem is antiretroviral (ARV) treatment was given to HIV-infected subpopulation, and $u_{2}(t)$ is HAART (highly active antiretroviral treatment) was given to full-blown AIDS subpopulation. The total population is divided into six compartments: $S(t), I_{1}(t), I_{2}(t)$, $A(t), T(t)$, and $R(t)$. $S(t)$ represents the number of susceptible individuals; $I_{1}(t)$ represents the number of HIV-positive individuals consuming ARV so that this subpopulation can survive longer; and $\mathrm{I}_{2}(\mathrm{t})$ represents the number of HIV-positive individuals not consuming ARV; $A(t)$ represents the number of individuals with full-blown AIDS not receiving treatment; $T(t)$ represents the number of individuals receiving ARV treatment; $R(t)$ represents the number of individuals who change their sexual habits and maintain the habits for the rest of their lives. This research is proposed to minimize the population with HIV infection and full-blown AIDS subpopulations, and the cost related to the implementation of control strategies. The functional objective can be defined as follows

$$
J\left(u_{1}(t), u_{2}(t)\right)=\int_{0}^{t f}\left(w_{1} u_{1}^{2}(t)+w_{2} u_{2}^{2}(t)+w_{3} I_{2}(t)+w_{4} A(t)\right) d t
$$


where $w_{i}, i=1,2,3,4$ represents weighted of ARV $\left(u_{1}\right)$, HAART $\left(u_{2}\right)$, infected subpopulation $\left(I_{2}\right)$, and full-blown AIDS $(A)$, respectively, $t_{f}$ is final time. We determine optimal control $u_{i}$ such that

$$
J\left(u_{i}^{*}(t)\right)=\min \left\{J\left(u_{i}(t)\right): u_{i}(t) \in U\right\}, i=1,2,
$$

with a set of a control function $U$ as follows

$$
\mathrm{U}=\left\{\mathrm{u}_{\mathrm{i}}(\mathrm{t}): 0 \leqslant \mathrm{u}_{\mathrm{i}}(\mathrm{t}) \leqslant 1, \mathrm{t} \in\left[0, \mathrm{t}_{\mathrm{f}}\right]\right\}, i=1,2 .
$$

In the next section, we will prove existence of optimal control by following $[6,16,19]$. We will prove the boundedness and super solution of system (4.1), where positivity of the solutions proved in [5].

\subsection{Invariant region}

The solution of system (4.1) with positive initial value will remain positive for all $t>0$, necessarily to be proved.

Theorem 4.1. All feasible sets $\mathrm{S}(\mathrm{t}), \mathrm{I}_{1}(\mathrm{t}), \mathrm{I}_{2}(\mathrm{t}), \mathrm{A}(\mathrm{t}), \mathrm{T}(\mathrm{t})$, and $\mathrm{R}(\mathrm{t})$ of system (4.1) are bounded by the region $\mathrm{D}=\left\{\left(\mathrm{S}, \mathrm{I}_{1}, \mathrm{I}_{2}, \mathrm{~A}, \mathrm{~T}, \mathrm{R}\right) \in \mathfrak{R}^{6}: \mathrm{S}+\mathrm{I}_{1}+\mathrm{I}_{2}+\mathrm{A}+\mathrm{T}+\mathrm{R} \leqslant \lambda / \mathrm{d}\right\}$.

Proof. From system equation (4.1),

$$
\dot{\mathrm{N}}=\dot{\mathrm{S}}+\dot{\mathrm{I}_{1}}+\dot{\mathrm{I}_{2}}+\dot{\mathrm{A}}+\dot{\mathrm{T}}+\dot{\mathrm{R}}=\lambda-\mathrm{dN}(\mathrm{t})-\delta_{1} \mathrm{~A}-\delta_{2} \mathrm{~T},
$$

implies that

$$
\dot{\mathrm{N}} \leqslant \lambda-\mathrm{dN}(\mathrm{t})
$$

and it follows that

$$
\mathrm{N} \leqslant \lambda / \mathrm{d}+\mathrm{N}(0) e^{-\mathrm{dt}},
$$

where $\mathrm{N}(0)$ is the initial value of total sub population. Thus

$$
\lim _{t \rightarrow \infty} \sup N(t) \leqslant \lambda / d,
$$

we end up $S+I_{1}+I_{2}+A+T+R \leqslant \lambda / d$. Hence for the analysis of the model (4.1), we get the region which is given by the set $D=\left\{\left(S, I_{1}, I_{2}, A, T, R\right) \in \mathfrak{R}^{6}: S+I_{1}+I_{2}+A+T+R \leqslant \lambda / d\right\}$, which is a positivity invariant set for system (4.1). We need to consider the dynamics of system (4.1) on non-negative solutions of the set D.

We agree that the super solutions of the system (4.1) are

$$
\begin{aligned}
\dot{S} & =\lambda, \\
\dot{\mathrm{I}_{1}} & =\beta_{1} \mathrm{SI}_{1}+\alpha_{1} \mathrm{~T}, \\
\dot{\mathrm{I}_{2}} & =\beta_{2} \mathrm{SI}_{2}, \\
\dot{\mathrm{A}} & =\mathrm{k}_{2} \mathrm{I}_{2}+\alpha_{2} \mathrm{~T}, \\
\dot{\mathrm{T}} & =\mathrm{k}_{1} \mathrm{I}_{1}+\mathrm{u}_{1} \mathrm{I}_{2}+\mathrm{u}_{2} A, \\
\dot{\mathrm{R}} & =\mu_{1} \mathrm{~S}+\mathrm{u}_{1} \mathrm{I}_{2},
\end{aligned}
$$

which are bounded on a finite time interval, and sub-solutions are zero.

\section{Existence of optimal control}

Theorem 5.1. Given the objective functional in (4.2), there exist an optimal control $u_{i}(t) * \in U, i=1,2$ such that the equation (4.2) satisfies the following conditions.

1. The control set $\mathrm{U}$ and the state variables $\left(\mathrm{S}(\mathrm{t}), \mathrm{I}_{1}(\mathrm{t}), \mathrm{I}_{2}(\mathrm{t}), \mathrm{A}(\mathrm{t}), \mathrm{T}(\mathrm{t})\right.$, and $\mathrm{R}(\mathrm{t})$ ), are not empty.

2. The control set $\mathrm{U}$ is convex and closed.

3. The right-hand side of the state system (4.1) is bounded above by linear function in the form state and control variables.

4. The integrand of the objective functional in (4.2) is convex on $\mathrm{U}$ and bounded below by $-v_{2}+v_{1} u_{i}^{2}$ with $v_{1}, v_{2}>0$. 
Proof.

1. By applying Theorems (4.1) and (5.1), the system (4.1) has bounded coefficients and the solutions on the finite time interval $0<\mathrm{t}<\infty$. To show the existence of the solution of the system (4.1), we can apply the result of Luxes (1982) [13].

2. The control set $U$ is closed and convex by definition.

3. The right-hand side of the system (4.1) must be continuous. The denominators of all fractions of the right-hand side of the system consists solely of positive entities. We let $\vec{G}(t, \vec{x})$ be right hand side of the system (4.1) without control variable

$$
\overrightarrow{\mathrm{F}}(\mathrm{t}, \overrightarrow{\mathrm{x}})=\overrightarrow{\mathrm{G}}(\mathrm{t}, \overrightarrow{\mathrm{x}})+\left[\begin{array}{c}
\lambda \\
0 \\
0 \\
0 \\
\mathrm{u}_{1} \mathrm{I}_{2}+\mathrm{u}_{2} A \\
\mathrm{u}_{1} \mathrm{I}_{2}
\end{array}\right]
$$

with $\vec{x}=\left[\begin{array}{llllll}S & I_{1} & I_{2} & A & T & R\end{array}\right]^{\top}$. Using the boundedness of the solutions, we get

$$
|\vec{F}(t, \vec{x})| \leqslant\left|\left[\begin{array}{cccccc}
0 & 0 & 0 & 0 & 0 & 0 \\
0 & \beta_{1} S & 0 & 0 & \alpha_{1} & 0 \\
0 & 0 & \beta_{2} S & 0 & 0 & 0 \\
0 & 0 & k_{2} & 0 & \alpha_{2} & 0 \\
0 & k_{1} & 0 & 0 & 0 & 0 \\
\mu_{1} & 0 & 0 & 0 & 0 & 0
\end{array}\right]\left[\begin{array}{c}
S \\
I_{1} \\
I_{2} \\
A \\
T \\
R
\end{array}\right]\right|+\left|\left[\begin{array}{c}
\lambda \\
0 \\
0 \\
0 \\
u_{1} I_{2}+u_{2} A \\
u_{1} I_{2}
\end{array}\right]\right| \leqslant v_{1}(|\vec{x}|+|\vec{u}|),
$$

where $v_{1}$ depends on the coefficients of the system. Therefore, the right side of the state equation is bounded from above by a sum of the state and control variable.

4. The integrand $h\left(u_{i}\right)$ of the functional $J$ is convex on $U$. The function $h\left(u_{i}\right)$ is convex in the interval $u_{i} \in[0,1], i=1,2$ when satisfies the following condition

$$
h\left(\theta u_{1}+(1-\theta) u_{2}\right) \leqslant \theta h\left(u_{1}\right)+(1-\theta) h\left(u_{2}\right) .
$$

For an arbitrary $\mathfrak{u}_{1}, \mathfrak{u}_{2} \in[0,1]$ with a given function

$$
h\left(u_{i}\right)=w_{1} u_{1}^{2}+w_{2} u_{2}^{2}+w_{3} I_{2}+w_{4} A,
$$

it is equivalent when we write the left hand side of equation (5.1) as follows

$$
h\left(\theta u_{1}+(1-\theta) u_{2}\right)=\left(w_{1}+w_{2}\right)\left(\theta u_{1}+(1-\theta) u_{2}\right)^{2}+w_{3} I_{2}+w_{4} A,
$$

and the right hand side of equation (5.1) is

$$
\theta h\left(u_{1}\right)+(1-\theta) h\left(u_{2}\right)=\left(w_{1}+w_{2}\right)\left(\theta u_{1}^{2}+(1-\theta) u_{2}^{2}\right)+w_{3} I_{2}+w_{4} A .
$$

By substituting equations (5.2) and (5.3) into (5.1), it yields

$$
\left(\theta u_{1}+(1-\theta) u_{2}\right)^{2} \leqslant\left(\theta u_{1}^{2}+(1-\theta) u_{2}^{2}\right) .
$$

By simple algebra we can write equation of the left-hand side of (5.4) as follows

$$
\left(\theta u_{1}+(1-\theta) u_{2}\right)^{2}=\left(\left(u_{1}-u_{2}\right) \theta\right)^{2}+u_{2}^{2}-2 u_{2}^{2} \theta\left(1-u_{1} / u_{2}\right),
$$


and the right-hand side of (5.4) as follows

$$
\left(\theta u_{1}^{2}+(1-\theta) u_{2}^{2}\right)=\theta u_{1}^{2}+u_{2}^{2}-\theta u_{2}^{2} .
$$

By choosing, $\theta \in[0,1]$, and $u_{1}, u_{2} \in[0,1]$, we agree $\left(\left(u_{1}-u_{2}\right) \theta\right)^{2} \leqslant \theta u_{1}^{2}$ and obviously $-2 u_{2}^{2} \theta\left(1-u_{1} / u_{2}\right) \leqslant$ $\left(-\theta u_{2}^{2}\right)$, hence equation (5.5) can be written as

$$
\left(\theta u_{1}+(1-\theta) u_{2}\right)^{2}=\left(\left(u_{1}-u_{2}\right) \theta\right)^{2}+u_{2}^{2}-2 u_{2}^{2} \theta\left(1-u_{1} / u_{2}\right) \leqslant \theta u_{1}^{2}+u_{2}^{2}-\theta u_{2}^{2},=\theta u_{1}^{2}+(1-\theta) u_{2}^{2} .
$$

From equation (5.6), we conclude that the integrand $h\left(u_{i}\right)$ of functional $J$ is convex on $U$.

Next, we will prove that integrand $h\left(u_{i}\right)$ of functional J is bounded below by $-v_{2}+v_{1} u_{i}^{2}$ with $v_{1}, v_{2}>0$, $i=1,2,3,4$ and $\vec{u}=u_{1}, u_{2}$. If there is $v_{1}>\epsilon / 2$ and remember $I_{2}$ and $A$ are bounded in the interval $[0,1]$ such that we have

$$
\mathrm{I}_{2}+\mathrm{A}+\frac{\epsilon}{2} \overline{\mathrm{u}}^{2} \geqslant \frac{\epsilon}{2} \overline{\mathrm{u}}^{2} \geqslant-v_{2}+\frac{\epsilon}{2} \mathrm{u}_{\mathrm{i}}^{2}
$$

which we can prove that the integrand $h\left(u_{i}\right)$ of functional $J$ is bounded from below by $-v_{2}+v_{1} u_{i}^{2}$.

Finally, we conclude there exists an optimal control of the system.

\section{The pontryagin minimum principle}

The optimality systems are solved by applying the Pontryagin-Minimum Principle as necessary condition. We introduce the state variables $S(t), I_{1}(t), I_{2}(t), A(t), T(t)$, and $R(t)$; and define the adjoint variables that corresponding to the state variables which are $\lambda_{1}(t), \lambda_{2}(t), \lambda_{3}(t), \lambda_{4}(t)$, and $\lambda_{5}(t)$. Thus, we introduce the Hamiltonian function as follows

$$
\mathrm{H}=\mathrm{h}+\sum_{i=1}^{5} \lambda_{i} g_{i},
$$

where $h$ is the integrand of functional objective (4.2) and $g_{i}$ is the right hand side of equation (4.1). The Hamiltonian of our problem is

$$
\begin{aligned}
H= & w_{1} u_{1}^{2}+w_{2} u_{2}^{2}+w_{3} I_{2}+w_{4} A+\lambda_{1}\left(\lambda-\beta_{1} S_{1}-\beta_{2} S_{2}-a S\right) \\
& +\lambda_{2}\left(\beta_{1} S_{1}+\alpha_{1} T-b I_{1}\right)+\lambda_{3}\left(\beta_{2} S_{2}-c_{1} I_{2}-u_{1} I_{2}\right) \\
& +\lambda_{4}\left(k_{2} I_{2}+\alpha_{2} T-e A-u_{2} A\right)+\lambda_{5}\left(k_{1} I_{1}+u_{1} I_{2}-f T+u_{2} A\right)+\lambda_{6}\left(\mu_{1} S-d R+u_{1} I_{2}\right)
\end{aligned}
$$

with $\lambda_{i}, i=1,2 \ldots, 6$ being known as co-state/adjoint variables. State equation is defined as following

$$
\frac{\partial H}{\partial \lambda_{i}}=g_{i}, \quad i=1,2,3, \ldots,
$$

with the initial conditions $S(0) \geqslant 0, I(0) \geqslant 0, A(0) \geqslant 0, T(0) \geqslant 0$, and $R(0) \geqslant 0$, and

$$
\begin{array}{lll}
\frac{\partial H}{\partial \lambda_{1}}=\lambda-\beta_{1} S I_{1}-\beta_{2} S I_{2}-a S, & \frac{\partial H}{\partial \lambda_{2}}=\beta_{1} S I_{1}+\alpha_{1} T-b I_{1}, \\
\frac{\partial H}{\partial \lambda_{3}}=\beta_{2} S I_{2}-c I_{2}-u_{1} I_{2}, & \frac{\partial H}{\partial \lambda_{4}}=k_{2} I_{2}+\alpha_{2} T-e A-u_{2} A, \\
\frac{\partial H}{\partial \lambda_{5}}=k_{1} I_{1}+u_{1} I_{2}-f T+u_{2} A, & \frac{\partial H}{\partial \lambda_{6}}=\mu_{1} S-d R+u_{1} I_{2} .
\end{array}
$$

Adjoint or co-state equation is defined as follows

$$
\frac{\mathrm{d} \lambda_{i}}{\mathrm{dt}}=-\frac{\partial \mathrm{H}}{\partial x_{i}}, \quad i=1,2,3, \ldots,
$$


such that we have

$$
\begin{aligned}
& \frac{\partial \lambda_{1}}{\partial t}=\lambda_{1}\left(\beta_{1} I_{1}+\beta_{2} I_{2}+a\right)-\lambda_{2} \beta_{1} I_{1}-\lambda_{3} \beta_{2} I_{2}-\lambda_{6} \mu_{1}, \\
& \frac{\partial \lambda_{2}}{\partial t}=\lambda_{1} \beta_{1} S+\lambda_{2}\left(b-\beta_{1} S\right)-\lambda_{5} k_{1}, \\
& \frac{\partial \lambda_{3}}{\partial t}=\lambda_{1} \beta_{2} S+\lambda_{3}\left(c+u_{1}-\beta_{2} S\right)-\lambda_{4} k_{2}-\lambda_{5} k_{3}-\lambda_{6} u_{1}-w_{3}, \\
& \frac{\partial \lambda_{4}}{\partial t}=\lambda_{4}\left(e+u_{2}\right)-\lambda_{5} u_{2}-w_{4}, \\
& \frac{\partial \lambda_{5}}{\partial t}=\lambda_{5} f-\lambda_{2} \alpha_{1}-\lambda_{4} \alpha_{2}, \\
& \frac{\partial \lambda_{6}}{\partial t}=\lambda_{6} d .
\end{aligned}
$$

Stationer condition is defined as follows

$$
\frac{\partial \mathrm{H}}{\partial u}=0,
$$

such that we obtain characteristic equation of the optimal control $u^{*}(t)$ as follows

$$
u_{1}^{*}(t)=\max \left\{\min \left\{\frac{\left(\lambda_{3}-\lambda_{6}\right) I_{2}}{2 w_{1}}, 1\right\}, 0\right\},
$$

and

$$
u_{2}^{*}(t)=\max \left\{\min \left\{\frac{\left(\lambda_{4}-\lambda_{5}\right) A}{2 w_{2}}, 1\right\}, 0\right\} .
$$

Therefore, the optimally system is given by

$$
\begin{aligned}
\frac{\partial H}{\partial \lambda_{1}} & =\lambda-\beta_{1} S^{*} I_{1}^{*}-\beta_{2} S^{*} I_{2}^{*}-a S^{*}, \\
\frac{\partial H}{\partial \lambda_{2}} & =\beta_{1} S^{*} I_{1}^{*}+\alpha_{1} T^{*}-b I_{1}^{*}, \\
\frac{\partial H}{\partial \lambda_{3}} & =\beta_{2} S^{*} I_{2}^{*}-c_{1} I_{2}-u_{1} I_{2}^{*}, \\
\frac{\partial H}{\partial \lambda_{4}} & =k_{2} I_{2}^{*}+\alpha_{2} T^{*}-e A^{*}-u_{2} A^{*}, \\
\frac{\partial H}{\partial \lambda_{5}} & =k_{1} I_{1}^{*}+u_{1}^{*} I_{2}^{*}-f T^{*}+u_{2} A^{*}, \\
\frac{\partial H}{\partial \lambda_{6}} & =\mu_{1} S^{*}-d R^{*}+u_{1}^{*} I_{2}^{*}, \\
\frac{\partial \lambda_{1}}{\partial t} & =\lambda_{1}\left(\beta_{1} I_{1}+\beta_{2} I_{2}^{*}+a\right)-\lambda_{2} \beta_{1} I_{1}^{*}-\lambda_{3} \beta_{2} I_{2}^{*}-\lambda_{6} \mu_{1}, \\
\frac{\partial \lambda_{2}}{\partial t} & =\lambda_{1} \beta_{1} S^{*}+\lambda_{2}\left(b-\beta_{1} S^{*}\right)-\lambda_{5} k_{1}, \\
\frac{\partial \lambda_{3}}{\partial t} & =\lambda_{1} \beta_{2} S^{*}+\lambda_{3}\left(c_{1}+u_{1}^{*}-\beta_{2} S^{*}\right)-\lambda_{4} k_{2}-\lambda_{5} u_{1}-\lambda_{6} u_{1}^{*}-w_{3}, \\
\frac{\partial \lambda_{4}}{\partial t} & =\lambda_{4}\left(e+u_{2}^{*}\right)-\lambda_{5} u_{2}^{*}-w_{4}, \\
\frac{\partial \lambda_{5}}{\partial t} & =\lambda_{5} f-\lambda_{2} \alpha_{1}-\lambda_{4} \alpha_{2}, \\
\frac{\partial \lambda_{6}}{\partial t} & =\lambda_{6} d, \\
u_{1}^{*}(t) & =\max \left\{\min \left\{\frac{\left(\lambda_{3}-\lambda_{6}\right) I_{2}^{*}}{2 w_{1}}, 1\right\}, 0\right\} \\
u_{2}^{*}(t) & =\max \left\{\min \left\{\frac{\left(\lambda_{4}-\lambda_{5}\right) A^{*}}{2 w_{2}}, 1\right\}, 0\right\} . \\
& =0,1(0) \geqslant 0, A(0) \geqslant 0, T(0) \geqslant 0, a n d R(0) \geqslant 0 .
\end{aligned}
$$

with the initial conditions $S(0) \geqslant 0, I(0) \geqslant 0, A(0) \geqslant 0, T(0) \geqslant 0$, and $R(0) \geqslant 0$. 


\section{Numerical simulation}

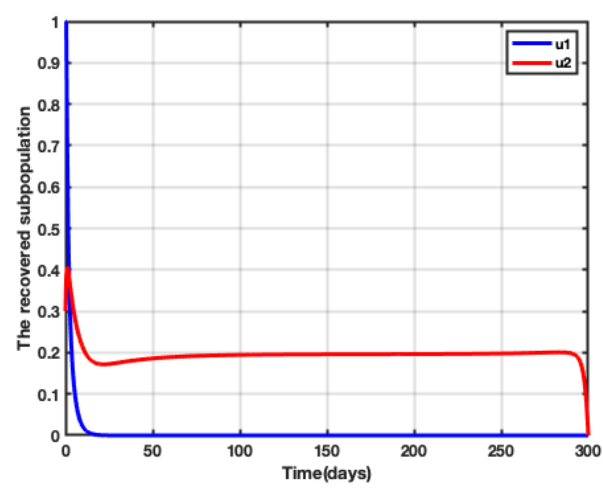

(a) Control profiles $u_{1}$ and $u_{2}$

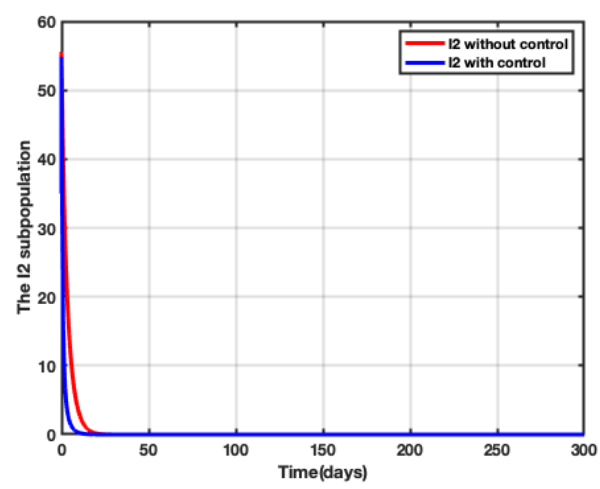

(c) $I_{2}$ subpopulation

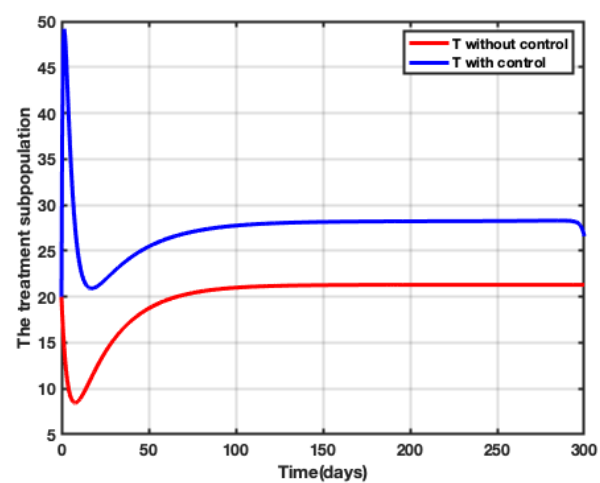

(e) T subpopulation

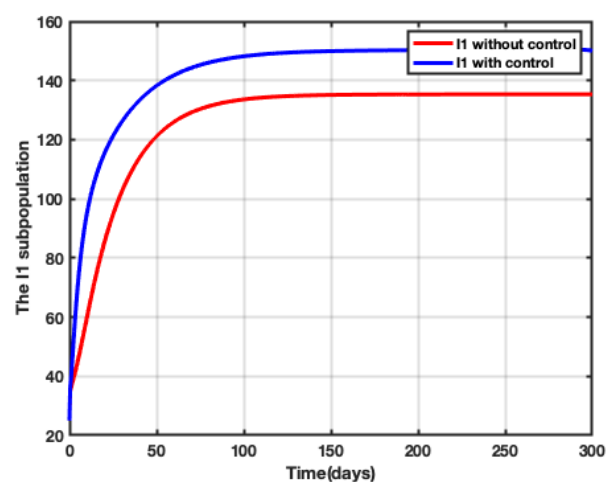

(b) $\mathrm{I}_{1}$ subpopulation

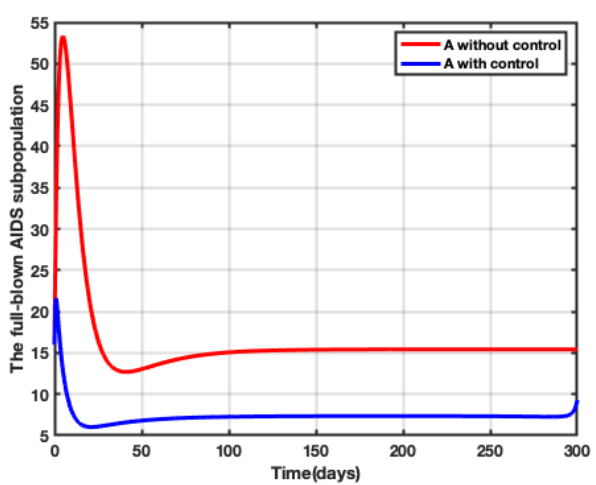

(d) A subpopulation

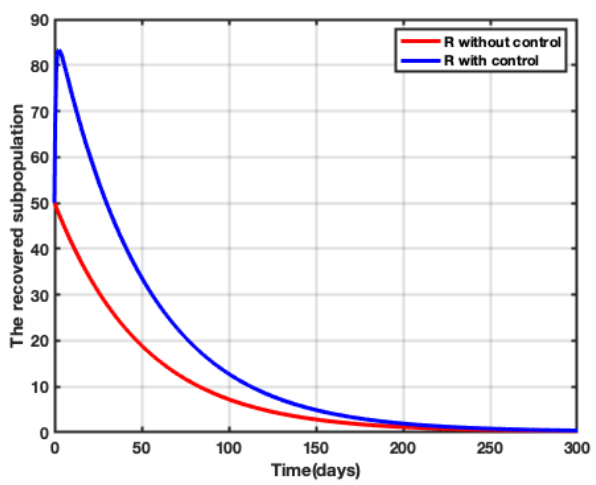

(f) R subpopulation

Figure 1: Numerical simulation of HIV/AIDS model, with and without controls $u_{1}$ and $u_{2}$. We used the data in Table 1 and $w_{1}=100$ and $w_{2}=100$ as the weights of controls $u_{1}$ and $u_{2}$, respectively.

To solve an optimally system numerically of equation (6.3), we apply forward and backward sweep method because equation (6.3) is in the form of the system of ordinary differential equation where it is difficult to solve analytically. We use the fourth order Runge-Kutta scheme since it has accuracy $\mathrm{O}\left(\mathrm{h}^{4}\right)$. The algorithm forward and backward sweep method is:

- Step 1: Choose an initial guess for a control $u_{i}^{*}$.

- Step 2: Solve the state system (4.1) by using a forward sweep loop with initial conditions in step 1. 
- Step 3: After obtaining the solution for the state system, we use backward loop to solve the co-states or adjoin systems (6.1) and (6.2) using transversal conditions.

- Step 4: Update a control $u_{i}^{*}$ in each iteration by using the values of the optimality system obtained in the previous iterations.

- Step 5: The procedure is continued iteratively till the convergence is achieved [11].

The simulation of all-related populated is observed before and after we give the ARV and HAART treatments as control strategies.

Figure 1 (a) shows control profile of $\mathfrak{u}_{1}$ and $\mathfrak{u}_{2}$. Control $\mathfrak{u}_{1}$ is given to infected individual initially 1 , then decreases to zero up to final time. Control $u_{2}$ is given to the full-blown AIDS initially 0.29, then increases to 0.43 at time 15 before decreases up to zero at the final time. Figure 1 (c) and (d) show that giving control can decrease the infected individual not-consuming ARV and AIDS subpopulation significantly, near to zero. Figure 1 (b), (e), and (f) show that giving control can increase the infected individual with consuming ARV, and treatment and recovered subpopulations increase significantly. From the numerical simulation, we can conclude that by giving ARV and HAART as control strategies in the model can decrease infected HIV and AIDS significantly.

\section{Conclusions}

We have studied the sensitivity and optimal control analysis of HIV/AIDS model. First, we show the sensitivity analysis of each parameter corresponds to the basic-reproduction number $\mathrm{R}_{0}$. The results showed that the most sensitive parameters to increase or decrease the basic reproduction number were the rate of recruitment $(\lambda)$ and the rate of transmission from uneducated subpopulations to infection not consuming ARVs $\left(\beta_{2}\right)$. Second, we have constructed optimal control problem of the epidemic model of HIV / AIDS. We proved boundedness and positivity of the solution, and existence of optimal control pair. The optimally system have been solved by applying the Minimum Pontryagin Principle to minimize the infected and full-blown AIDS subpopulations, and the cost related to the implementation of control pair strategies. Numerical solutions are conducted to solve the optimally system using the sweep backward and forward method. The results show that by giving ARV and HAART can decrease the infected and the full-blown AIDS subpopulations significantly.

\section{Acknowledgment}

The authors would like to thank Brawijaya University for supporting an author trough Hibah Doktor 2020, No: 49/UN10/F09/PN/2020.

\section{References}

[1] W. Assawinchaichote, Robust $\mathrm{H}_{\infty}$ Controller Design for Nonlinear Positive HIV/AIDS Infection Dynamic Model: A Fuzzy Approach, Proc. Int. MultiConf. Eng. Comput. Sci., I (2012), 5 pages. 1

[2] L. M. Cai, S. L. Guo, S. P. Wang, Analysis of an extended HIV/AIDS epidemic model with treatment, Appl. Math. Comput., 236 (2014), 621-627. 1

[3] L. M. Cai, X. Z. Li, M. Ghosh, B. Z. Guo, Stability analysis of an HIV/AIDS epidemic Model with treatment, J. Comput. Appl. Math., 229 (2009), 313-323. 1

[4] N. Chitnis, J. M. Hyman, J. M. Cushing, Determining important parameters in the spread of malaria through the sensitivity analysis of a mathematical model, Bull. Math. Biol., 70 (2008), 1272-1296. 1

[5] H. R. Erfanian, M. H. Noori Skandari, Optimal Control of an HIV Model, J. Math. Comput. Sci., 2 (2011), $650-658$. $1,3,4$

[6] U. Habibah, R. A. Sari, Optimal control analysis of HIV/AIDS epidemic model with an antiretroviral treatment, Aust. J. Math. Anal. Appl., 17 (2020), 11 pages. 1, 4

[7] U. Habibah, R. A. Sari, The Effectiveness of an Antiretroviral Treatment (ARV) and a Highly Active Antiretroviral Theraphy (HAART) on HIV/AIDS Epidemic Model, AIP Conference Proceedings, 2021 (2018), 12 pages. 1 
[8] U. Habibah, Trisilowati, Y. L. Pradana, W. Villadistyan, Mathematical model of HIV/AIDS with two different stages of infection subpopulation and its stability analysis, Eng. Lett., 29 (2020), 13 pages. 1, 2, 1, 4, 4

[9] H.-F. Huo, R. Chen, Stability of an HIV/AIDS Treatment Model with Different Stages, Discrete Dyn. Nat. Soc., 2015 (2015), 9 pages. 1

[10] H.-F. Huo, R. Chen, X.-Y. Wang, Modelling and Stability of HIV/AIDS Epidemic Model with Treatment, Appl. Math. Model., 40 (2016), 6550-6559. 1

[11] S. Khajanchi, D. Ghosh, The Combined Effects of Optimal Control in Cancer Remission, Appl. Math. Comput., 271 (2015), 375-388. 1, 7

[12] S. Lenhart, J. T. Workman, Optimal Control Applied to Biological Models, Chapman \& Hall/CRC, Boca Raton, (2007). 1

[13] D. L. Lukes, Differential Equations: Clasical to controlled, Academic Press, New York, (1982). 5

[14] M. Marsudi, N. Hidayat, R. B. E. Wibowo, Application of Optimal Control Strategies for the Spread of HIV in a Population, Res. J. Life Sci., 4 (2017), 9 pages. 1

[15] M. Najariyan, M. H. Farahi, M. Alavian, Optimal Control of HIV Infection by using Fuzzy Dynamical Systems, J. Math. Comput. Sci., 2 (2011), 639-649. 1

[16] S. I. Oke, M. B. Matadi, S. S. Xulu, Optimal Control Analysis of a Mathematical Model for Breast Cancer, Math. Comput. Appl., 23 (2018), 28 pages. 4

[17] M. A. Rois, T. Trisilowati, U. Habibah, Local Sensitivity Analysis of COVID-19 Epidemic with Quarantine and Isolation using Normalized Index, Telematika, 14 (2021), 13-24. 1, 3, 3

[18] S. Saha, G. P. Samanta, Modelling and Optimal Control of HIV/AIDS prevention through PrEP and Limited Treatment, Phys. A, 516 (2019), 280-307. 1

[19] T. Trisilowati, Mathematical Modelling of Tumor Growth and Interaction with Host Tissue and the Immune System, PhD thesis, Mathematical Sciences School, Queensland University of Technology, (2012). 4

[20] B. Ulfa, T. Trisilowati, W. M. Kusumawinahyu, Dynamical Analysis of HIV/AIDS Epidemic Model with Treatment, J. Exp. Life Sci., 8 (2018), 11 pages. 1

[21] S. Mushayabasa, C.P. Bhunu, Modeling HIV Transmission Dynamics among Male prisoners in Sub-Saharan Africa, IAENG Inter. J. Appl. Math., 41 (2011), 6 pages. 1 